International Mathematical Forum, Vol. 9, 2014, no. 6, 291 - 303

HIKARI Ltd, www.m-hikari.com

http://dx.doi.org/10.12988/imf.2014.4118

\title{
Some Simple Properties and Existence of HS-Integrals in a Locally Convex Space
}

\author{
Juniven P. Acapulco ${ }^{1}$ \\ Mathematics Department \\ College of Natural Sciences and Mathematics \\ MSU-General Santos \\ General Santos City, Philippines \\ Julius V. Benitez \\ Mathematics Department \\ College of Science and Mathematics \\ MSU-Iligan Institute of Technology \\ Iligan City, Philippines
}

Copyright (c) 2014 Juniven P. Acapulco and Julius V. Benitez. This is an open access article distributed under the Creative Commons Attribution License, which permits unrestricted use, distribution, and reproduction in any medium, provided the original work is properly cited.

\begin{abstract}
In this paper, we define the Henstock-Stieltjes integral for functions that take values in a locally convex space. Results such as uniqueness, linearity, addidivity, Cauchy Criterion, and integrability on subintervals are presented. Moreover, some conditions on the existence of the Henstock-Stieltjes integrals are also presented.
\end{abstract}

Keywords: Locally convex topological vector space, Henstock-Stieltjes integral

\footnotetext{
${ }^{1}$ Research supported in part by the Department of Science and Technology-Accelerated Science and Technology Human Resource Development Program (DOST-ASTHRDP), Philippines
} 


\section{Introduction}

Many definitions of Stieltjes-type integrals have been proposed since the original one by T.J. Stieltjes in 1894 [6]. For instance, the Henstock-Stieltjes integral for real-valued functions was defined and investigated in 1998 by Lim, Yoon, and Eun [2]. In this note, we will discuss a locally convex space-valued version of the Henstock-Stieltjes integral. For Banach-valued approach, see [5]. The motivation of this study is based on the work of Marraffa [3].

Althroughout this paper, $(X, \tau)$ is a Hausdorff locally convex topological vector space (briefly a locally convex space) and $\mathcal{P}(X)$ is a separating family of $\tau$-continuous semi-norms on $X$ that generates the topology $\tau$. The space $X$ is called complete if every Cauchy net in $X$ converges. A family $\Gamma$ of semi-norms on a vector space $X$ has the max-property if for any $p_{1}, p_{2} \in \Gamma$ there exists $p \in \Gamma$ and $c>0$ such that $p \geq c \max \left\{p_{1}, p_{2}\right\}$.

Let $-\infty<a<b<+\infty$. Then any function $\delta:[a, b] \rightarrow(0,+\infty)$ is often called a gauge on $[a, b]$. For a gauge $\delta$ on $[a, b]$, a division $D=\{([u, v], t)\}$ of $[a, b]$ is called a $\delta$-fine division if $t \in[u, v] \subset(t-\delta(t), t+\delta(t))$ for each interval-point pair $([u, v], t)$. For an arbitrary gauge $\delta$ on $[a, b]$, Cousin's lemma guarantees the existence of a $\delta$-fine division on $[a, b]$, see [7]. Let $\delta$ be a gauge on $[a, b]$. Let $D$ and $D^{\prime}$ be $\delta$-fine divisions of $[a, b]$. We say that $D^{\prime}$ is finer than $D$ (or $D^{\prime}$ is a refinement of $D$ ) if for every $\left(\left[u^{\prime}, v^{\prime}\right], t^{\prime}\right) \in D^{\prime}$ there exists $([u, v], t) \in D$ such that $\left[u^{\prime}, v^{\prime}\right] \subset[u, v]$ and every tag of $D$ is a tag of $D^{\prime}$.

Let $f:[a, b] \rightarrow X$ and $t \in(a, b)$. The function $f$ has a limit at $t$ if there exists $\alpha \in X$ such that $\forall p \in \mathcal{P}(X)$ and $\forall \epsilon>0$, we can find $\delta>0$ such that for any $x \in[a, b]$ with $0<|x-t|<\delta$, we have $p(f(x)-\alpha)<\epsilon$. In this case, we write

$$
\lim _{x \rightarrow t} f(x)=\alpha \quad \text { or } \quad f(x) \rightarrow \alpha \text { as } x \rightarrow t .
$$

Let $f:[a, b] \rightarrow X$ and $t \in[a, b)$ (resp. $t \in(a, b]$ ). The function $f$ has a right limit (resp. left limit) at $t$ if there exists $\alpha \in X$ such that $\forall p \in \mathcal{P}(X)$ and $\forall \epsilon>0$, we can find $\delta>0$ such that for any $x \in[a, b]$ with $t<x<t+\delta$ (resp. $t-\delta<x<t$ ), we have $p(f(x)-\alpha)<\epsilon$. The right limit (resp. left limit) of $f$ at $t$, if it exists, is denoted by $f(t+)$ (resp. $f(t-)$ ), and we write

$$
f(t+)=\lim _{x \rightarrow t^{+}} f(x) \quad\left(\operatorname{resp} . f(t-)=\lim _{x \rightarrow t^{-}} f(x)\right) .
$$

A function $f:[a, b] \rightarrow X$ is said to be continuous at $t \in[a, b]$ if for every $p \in \mathcal{P}(X)$ and $\epsilon>0$ there exists $\delta>0$ such that for all $x \in[a, b]$ with $|x-t|<\delta$, we have $p(f(x)-f(t))<\epsilon$. The function $f:[a, b] \rightarrow X$ is said to becontinuous on $[a, b]$ if it is continuous at every point of $[a, b]$. A function $f:[a, b] \rightarrow X$ is said to be uniformly continuous if for every $p \in \mathcal{P}(X)$ and $\epsilon>0$ there exists $\delta>0$ such that for all $x, y \in[a, b]$ with $|x-y|<\delta$, we have 
$p(f(x)-f(y))<\epsilon$. A function $f:[a, b] \rightarrow X$ is said to be bounded if for every $p \in \mathcal{P}(X)$, there exists $M>0$ such that $p(f(t)) \leq M$ for all $t \in[a, b]$.

A function $f:[a, b] \rightarrow X$ is said to be regulated on $[a, b]$ if it has one sided limits at every point of $[a, b]$, that is, for every $t \in[a, b), f(t+)$ exists and for every $t \in(a, b], f(t-)$ exists. The set of all regulated functions $f:[a, b] \rightarrow X$ will be denoted by $G([a, b], X)$. A function $\psi:[a, b] \rightarrow X$ is said to be a step function on $[a, b]$ if there exists a division $D=\left\{\left[x_{i-1}, x_{i}\right]\right\}_{i=1}^{n}$ of $[a, b]$ such that $\psi$ has a constant value in $X$ on $\left(x_{i-1}, x_{i}\right)$ for every $i=1, \ldots, n$. Let $\left(f_{n}\right)$ be a sequence of $X$-valued functions defined on $[a, b]$ and let $f:[a, b] \rightarrow X$. We say that $\left(f_{n}\right)$ converges uniformly to $f$ on $[a, b]$ if for every $p \in \mathcal{P}(X)$ and $\epsilon>0$ there exists a positive integer $N$ (independent of $x$ ) such that for all $x \in[a, b]$ and for all $n \geq N$, we have $p\left(f_{n}(x)-f(x)\right)<\epsilon$. With the preceding definitions, it can be shown that a function $f:[a, b] \rightarrow X$ is regulated iff there exists a sequence $\left(\psi_{n}\right)$ of step functions defined on $[a, b]$ that converges uniformly to $f$ on $[a, b]$.

A function $g:[a, b] \rightarrow X$ is said to be of bounded variation on $[a, b]$ if for each $p \in \mathcal{P}(X)$,

$$
T V_{a}^{b}(g)_{p}=\sup \left\{\sum_{i=1}^{n} p\left(g\left(x_{i}\right)-g\left(x_{i-1}\right)\right)\right\}<\infty
$$

where the supremum is taken over all divisions $D=\left\{\left[x_{i-1}, x_{i}\right]\right\}_{i=1}^{n}$ of $[a, b]$. The set of all functions $g:[a, b] \rightarrow X$ which are of bounded variations will be denoted by $B V([a, b], X)$.

\section{Some Basic Properties}

In what follows, $\mathbb{R}$ denotes the set of reals, $\mathbb{N}$ the set of all positive integers and $\theta$ is the zero vector in $X$.

Definition 2.1. Let $f:[a, b] \rightarrow X($ resp. $\mathbb{R})$ and $g:[a, b] \rightarrow \mathbb{R}($ resp. $X)$ be any two functions. We say that the Henstock-Stieltjes integral of $f$ with respect to $g$ on $[a, b]$ exists with value $w \in X$, and write $w=(H S) \int_{a}^{b} f d g$, if given $\epsilon>0$ and $p \in \mathcal{P}(X)$ there exists a gauge $\delta_{p}$ on $[a, b]$ such that for all $\delta_{p}$-fine division $D=\{([u, v], t)\}$ of $[a, b]$, we have $p(S[f, g, D]-w)<\epsilon$, where $S[f, g, D]=(D) \sum f(t)[g(v)-g(u)]$ is called the Henstock-Stieltjes sum of $f$ with respect to $g$ over $D$. In this case $w$ is called the Henstock-Stieltjes integral of $f$ with respect to $g$ over $[a, b]$. We also say that $(f, g)$ is Henstock-Stieltjes integrable (or simply $H S$-integrable) to $w$ on $[a, b]$. The function $f$ is usually called the integrand and $g$ the integrator. 
Theorem 2.2. There is at most one vector $w$ satisfying the condition from Definition 2.1.

Proof. Let $w_{1}$ and $w_{2}$ be two vectors satisfying Definition 2.1. Let $\epsilon>0$ and $p \in \mathcal{P}(X)$. Then there exist gauges $\delta_{p}^{i}, i=1,2$, on $[a, b]$ such that for all $\delta_{p}^{i}$-fine division $D=\{([u, v], t)\}$ of $[a, b], p\left(S[f, g, D]-w_{i}\right)<\frac{\epsilon}{2}$.

Define $\delta_{p}=\min \left\{\delta_{p}^{1}, \delta_{p}^{2}\right\}$. Then $\delta_{p}$ is a gauge on $[a, b]$ and hence by the Cousin's Lemma, there exists a $\delta_{p}$-fine division $D=\{([u, v], t)\}$ of $[a, b]$. Clearly, $D$ is both $\delta_{p}^{1}$-fine and $\delta_{p}^{2}$-fine. Thus,

$$
p\left(w_{1}-w_{2}\right) \leq p\left(S[f, g, D]-w_{1}\right)+p\left(S[f, g, D]-w_{2}\right)<\frac{\epsilon}{2}+\frac{\epsilon}{2}=\epsilon .
$$

From the arbitrariness of $\epsilon>0$, we get $p\left(w_{1}-w_{2}\right)=0$ for all $p \in \mathcal{P}(X)$. Since $\mathcal{P}(X)$ is separating, it follows that $w_{1}-w_{2}=\theta$. Hence, $w_{1}=w_{2}$.

Theorem 2.3. If $f$ is $H S$-integrable with respect to both $g_{1}$ and $g_{2}$ on $[a, b]$, then for any $c_{1}, c_{2} \in \mathbb{R}, f$ is $H S$-integrable with respect to $c_{1} g_{1}+c_{2} g_{2}$ on $[a, b]$ and

$$
(H S) \int_{a}^{b} f d\left(c_{1} g_{1}+c_{2} g_{2}\right)=c_{1} \cdot(H S) \int_{a}^{b} f d g_{1}+c_{2} \cdot(H S) \int_{a}^{b} f d g_{2} .
$$

Proof. For $i=1,2$, we let $w_{i}=(H S) \int_{a}^{b} f d g_{i}$. Let $\epsilon>0$ and $p \in \mathcal{P}(X)$. Then there exist gauges $\delta_{p}^{i}, i=1,2$, on $[a, b]$ such that for all $\delta_{p}^{i}$-fine division $D=\{([u, v], t)\}$ of $[a, b]$,

$$
p\left(S\left[f, g_{i}, D\right]-w_{i}\right)<\frac{\epsilon}{2\left(1+\left|c_{i}\right|\right)} .
$$

Define $\delta_{p}=\min \left\{\delta_{p}^{1}, \delta_{p}^{2}\right\}$. Then $\delta_{p}$ is a gauge on $[a, b]$. Let $D=\{([u, v], t)\}$ be a $\delta_{p}$-fine division of $[a, b]$. Clearly, $D$ is both $\delta_{p}^{1}$-fine and $\delta_{p}^{2}$-fine. Thus,

$$
\begin{aligned}
p(S[f & \left.\left., c_{1} g_{1}+c_{2} g_{2}, D\right]-\left[c_{1} w_{1}+c_{2} w_{2}\right]\right) \\
& \leq p\left(S\left[f, c_{1} g_{1}, D\right]-c_{1} w_{1}\right)+p\left(S\left[f, c_{2} g_{2}, D\right]-c_{2} w_{2}\right) \\
& =\left|c_{1}\right| \cdot p\left(S\left[f, g_{1}, D\right]-w_{1}\right)+\left|c_{2}\right| \cdot p\left(S\left[f, g_{2}, D\right]-w_{2}\right) \\
& <\frac{\left|c_{1}\right| \epsilon}{2\left(1+\left|c_{1}\right|\right)}+\frac{\left|c_{2}\right| \epsilon}{2\left(1+\left|c_{2}\right|\right)}<\frac{\epsilon}{2}+\frac{\epsilon}{2}=\epsilon .
\end{aligned}
$$

Using Definition 2.1, the result follows.

Theorem 2.4. If $f_{1}$ and $f_{2}$ are both $H S$-integrable with respect to $g$ on $[a, b]$, then for any $c_{1}, c_{2} \in \mathbb{R}, c_{1} f_{1}+c_{2} f_{2}$ is $H S$-integrable with respect to $g$ on $[a, b]$ and

$$
(H S) \int_{a}^{b}\left(c_{1} f_{1}+c_{2} g_{2}\right) d g=c_{1} \cdot(H S) \int_{a}^{b} f_{1} d g+c_{2} \cdot(H S) \int_{a}^{b} f_{2} d g .
$$


Proof. Using the same arguments in the proof of Theorem 2.3 and the fact that $S\left[c_{1} f_{1}+c_{2} f_{2}, g, D\right]=c_{1} \cdot S\left[f_{1}, g, D\right]+c_{2} \cdot S\left[f_{2}, g, D\right]$, the result follows.

Theorem 2.5. Let $a<c<b$. If $f$ is both $H S$-integrable with respect to $g$ on $[a, c]$ and $[c, b]$, then $f$ is $H S$-integrable with respect to $g$ on $[a, b]$. Moreover,

$$
(H S) \int_{a}^{b} f d g=(H S) \int_{a}^{c} f d g+(H S) \int_{c}^{b} f d g .
$$

Proof. Let $(H S) \int_{a}^{c} f d g=w_{1}$ and $(H S) \int_{c}^{b} f d g=w_{2}$. Let $\epsilon>0$ and $p \in \mathcal{P}(X)$. Then there exists a gauge $\delta_{p}^{1}$ on $[a, c]$ such that for every $\delta_{p^{-}}^{1}$ fine division $D_{1}$ of $[a, c]$, we have $p\left(S\left[f, g, D_{1}\right]-w_{1}\right)<\frac{\epsilon}{2}$. Also, there exists a gauge $\delta_{p}^{2}$ on $[c, b]$ such that for every $\delta_{p}^{2}$-fine division $D_{2}$ of $[c, b]$, we have $p\left(S\left[f, g, D_{2}\right]-w_{2}\right)<\frac{\epsilon}{2}$. For each $t \in[a, b]$, let

$$
\delta_{p}(t)= \begin{cases}\min \left\{\delta_{p}^{1}(t), c-t\right\}, & \text { if } t \in[a, c) \\ \min \left\{\delta_{p}^{1}(t), \delta_{p}^{2}(t)\right\}, & \text { if } t=c \\ \min \left\{\delta_{p}^{2}(t), t-c\right\}, & \text { if } t \in(c, b] .\end{cases}
$$

This defines a gauge $\delta_{p}$ on $[a, b]$. Let $D=\left\{\left(\left[x_{i-1}, x_{i}\right], t_{i}\right)\right\}_{i=1}^{n}$ be a $\delta_{p}$-fine division of $[a, b]$. Then we can find $k \in\{1,2, \ldots, n\}$ such that $t_{k}=c$. Thus, it follows that there exists $k \in\{1,2, \ldots, n\}$ such that $c$ is a tag of $\left[x_{k-1}, x_{k}\right]$. Set

$$
D_{1}=\left\{\left(\left[x_{i-1}, x_{i}\right], t_{i}\right)\right\}_{i=1}^{k-1} \cup\left\{\left(\left[x_{k-1}, c\right], c\right)\right\}
$$

and

$$
D_{2}=\left\{\left(\left[c, x_{k}\right], c\right)\right\} \cup\left\{\left(\left[x_{i-1}, x_{i}\right], t_{i}\right)\right\}_{i=k+1}^{n} .
$$

Clearly, $D=D_{1} \cup D_{2}$. Note that $\left.\left\{\left[x_{k-1}, c\right], c\right)\right\}$ is $\delta_{p}^{1}$-fine and $\left.\left\{\left[c, x_{k}\right], c\right)\right\}$ is $\delta_{p}^{2}$-fine. Thus $D_{1}$ and $D_{2}$ are $\delta_{p}^{1}$-fine and $\delta_{p}^{2}$-fine divisions of $[a, c]$ and $[c, b]$, respectively. Thus,

$$
\begin{array}{r}
p\left(S[f, g, D]-\left[w_{1}+w_{2}\right]\right)=p\left(S\left[f, g, D_{1}\right]+S\left[f, g, D_{2}\right]-w_{1}-w_{2}\right) \\
\leq p\left(S\left[f, g, D_{1}\right]-w_{1}\right)+p\left(S\left[f, g, D_{2}\right]-w_{2}\right)<\frac{\epsilon}{2}+\frac{\epsilon}{2}=\epsilon
\end{array}
$$

Therefore, by Definition 2.1, the result follows.

The next result is useful when there is no particular value that can be predicted to be the HS-integral of a function. We will see later that it has some useful consequences. The idea of the proof is borrowed from [3].

Theorem 2.6. Let $X$ be a complete LCS and suppose that $\mathcal{P}(X)$ has the max-property. Then the following are equivalent. 
(i) $f$ is HS-integrable with respect to $g$ on $[a, b]$.

(ii) For every $\epsilon>0$ and $p \in \mathcal{P}(X)$ there exists a gauge $\delta_{p}$ on $[a, b]$ such that for all $\delta_{p}$-fine divisions $D_{1}$ and $D_{2}$ of $[a, b], p\left(S\left[f, g, D_{1}\right]-S\left[f, g, D_{2}\right]\right)<\epsilon$.

Proof. $(i) \Rightarrow(i i)$. Suppose that $w=(H S) \int_{a}^{b} f d g$ exists. Let $\epsilon>0$ and $p \in \mathcal{P}(X)$. Then there exists a gauge $\delta_{p}$ such that if $D_{1}$ and $D_{2}$ are $\delta_{p}$-fine divisions of $[a, b]$, then

$$
p\left(S\left[f, g, D_{1}\right]-w\right)<\frac{\epsilon}{2} \quad \text { and } \quad p\left(S\left[f, g, D_{2}\right]-w\right)<\frac{\epsilon}{2} .
$$

Consequently, we conclude that for such divisions $D_{1}$ and $D_{2}$, then

$$
\begin{aligned}
p\left(S\left[f, g, D_{1}\right]-S\left[f, g, D_{2}\right]\right) & \leq p\left(S\left[f, g, D_{1}\right]-w\right)+p\left(S\left[f, g, D_{2}\right]-w\right) \\
& \leq \frac{\epsilon}{2}+\frac{\epsilon}{2}=\epsilon .
\end{aligned}
$$

$(i i) \Rightarrow(i)$. Assume that statement $(i i)$ holds. Let us work with the functions $f:[a, b] \rightarrow X$ and $g:[a, b] \rightarrow \mathbb{R}$. Define the set

$$
\mathbb{D}=\{(K, m): K \text { is a finite subset of } \mathcal{P}(X) \text { and } m \in \mathbb{N}\} .
$$

It can be shown that $\mathbb{D}$ is a directed set with respect to the partial order $\leqq$ defined as follows: For all $(K, m),(L, n) \in \mathbb{D},(K, m) \leqq(L, n)$ iff $K \subset L$ and $m \leq n$. We shall now construct a net on the space $X$. Let $(K, m) \in \mathbb{D}$. Then we can write $K=\left\{p_{1}, p_{2}, \ldots, p_{k}\right\}$ for some $p_{1}, p_{2}, \ldots, p_{k} \in \mathcal{P}(X)$. Since $\mathcal{P}(X)$ has the max-property, we can find $p_{K} \in \mathcal{P}(X)$ and a constant $C_{K}>0$ such that the inequality $p_{i} \leq C_{K} \cdot p_{K}$ for $i=1,2, \ldots, k$ holds on $X$. Corresponding to $m$ and for each $i \in\{1,2, \ldots, k\}$, we can find a gauge $\delta_{p_{i}}^{m}$ on $[a, b]$ such that for all $\delta_{p_{i}}^{m}$-fine divisions $D_{1}$ and $D_{2}$ of $[a, b]$,

$$
p_{i}\left(S\left[f, g, D_{1}\right]-S\left[f, g, D_{2}\right]\right)<\frac{1}{m} .
$$

Also, there exists a gauge $\delta_{K}^{m}$ such that for all $\delta_{K}^{m}$-fine divisions $D_{1}$ and $D_{2}$ of $[a, b]$,

$$
p_{K}\left(S\left[f, g, D_{1}\right]-S\left[f, g, D_{2}\right]\right)<\frac{1}{m \cdot C_{K}} .
$$

WLOG, assume that $\delta_{K}^{m} \leq \min \left\{\delta_{p_{1}}^{m}, \delta_{p_{2}}^{m}, \ldots, \delta_{p_{k}}^{m}\right\}$. Moreover, we can assume that for each fixed finite set $K \subset \mathcal{P}(X)$, the sequence $\left(\delta_{K}^{m}\right)$ is nondecreasing. Since $\delta_{K}^{m}$ is a gauge on $[a, b]$, it follows from Cousin's Lemma that there is a $\delta_{K}^{m}$-fine division $D_{K}^{m}$ of $[a, b]$. Then we form the sum $S\left[f, g, D_{K}^{m}\right] \in X$. We denote this unique sum by $x_{(K, m)}$. This defines a net $\left(x_{(K, m)}\right)_{(K, m) \in \mathbb{D}}$.

Claim. The net $\left(x_{(K, m)}\right)_{(K, m) \in \mathbb{D}}$ is a Cauchy net. 
Let $\eta>0$ and $p \in \mathcal{P}(X)$. Choose $N \in \mathbb{N}$ such that $\frac{1}{N}<\frac{\eta}{2}$. Clearly, $(\{p\}, N) \in \mathbb{D}$. Assume that $(K, m),(L, n) \geqq(\{p\}, N)$. Then $\{p\} \subset K, L$ and $m, n \geq N$. Consider a $\delta_{p}^{N}$-fine division $D_{p}^{\bar{N}}$ of $[a, b]$. Because $m, n \geq N$, it follows that

$$
\delta_{p}^{N} \leq \delta_{p}^{m} \quad \text { and } \quad \delta_{p}^{N} \leq \delta_{p}^{n}
$$

hold on $[a, b]$. Thus, $D_{p}^{N}$ is both $\delta_{p}^{m}$-fine and $\delta_{p}^{n}$-fine. Next, we consider $\delta_{K}^{m}$-fine and $\delta_{K}^{n}$-fine divisions $D_{K}^{m}$ and $D_{K}^{n}$ of $[a, b]$, respectively. Since $p \in K \cap L$, it follows that

$$
\delta_{K}^{m} \leq \delta_{p}^{m} \quad \text { and } \quad \delta_{L}^{n} \leq \delta_{p}^{n}
$$

hold on $[a, b]$. Hence, $D_{K}^{m}$ is $\delta_{p}^{m}$-fine and $D_{L}^{n}$ is $\delta_{p}^{n}$-fine. Thus,

$$
\begin{aligned}
& p\left(x_{(K, m)}-x_{(L, n)}\right)=p\left(S\left[f, g, D_{K}^{m}\right]-S\left[f, g, D_{K}^{n}\right]\right) \\
& \quad \leq p\left(S\left[f, g, D_{K}^{m}\right]-S\left[f, g, D_{p}^{N}\right]\right)+p\left(S\left[f, g, D_{p}^{N}\right]-S\left[f, g, D_{K}^{n}\right]\right) \\
& \quad<\frac{1}{m}+\frac{1}{n} \leq \frac{1}{N}+\frac{1}{N}<\eta .
\end{aligned}
$$

Since $p$ was arbitrary, the claim follows.

By completeness of $X$, it follows that the net $\left(x_{(K, m)}\right)_{(K, m) \in \mathbb{D}}$ converges to some $w \in X$. We show that $(H S) \int_{a}^{b} f d g=w$. Let $\epsilon>0$ and $q \in \mathcal{P}(X)$. Then there exists $\left(K_{1}, N_{1}\right) \in \mathbb{D}$ such that for all $(K, m) \geqq\left(K_{1}, N_{1}\right)$,

$$
q\left(x_{(K, m)}-w\right)<\frac{\epsilon}{2}
$$

Also, we choose $N_{2} \in \mathbb{N}$ such that for all $(K, m),(L, n) \geqq\left(\{q\}, N_{2}\right)$,

$$
q\left(x_{(K, m)}-x_{(L, n)}\right)<\frac{\epsilon}{2}
$$

Choose $(S, N) \in \mathbb{D}$ such that $\left(\{q\}, N_{2}\right) \leqq(S, N)$ and $\left(K_{1}, N_{1}\right) \leqq(S, N)$. Corresponding to $(S, N) \in \mathbb{D}$, a gauge $\delta_{S}^{N}$ exists. Let $D$ be a $\delta_{S}^{N}$-fine division of $[a, b]$. Choose any $(K, m) \in \mathbb{D}$ such that $(K, m) \geqq(S, N)$. Hence,

$$
\begin{aligned}
q(S[f, g, D]-w) & \leq q\left(S[f, g, D]-S\left[f, g, D_{K}^{m}\right]\right)+q\left(S\left[f, g, D_{K}^{m}\right]-w\right) \\
& =q\left(x_{(S, N)}-x_{(K, m)}\right)+q\left(x_{(K, m)}-w\right) \\
& <\frac{\epsilon}{2}+\frac{\epsilon}{2}=\epsilon .
\end{aligned}
$$

It follows from Definition 2.1 that $f$ is HS-integrable with respect to $g$ on $[a, b]$.

We now consider one application of the Cauchy Criterion. This result tells us that the HS-integral can be considered as a function of intervals. 
Theorem 2.7. Let $X$ be a complete LCS and suppose that $\mathcal{P}(X)$ has the max-property. If $f$ is $H S$-integrable with respect to $g$ on $[a, b]$, then for every $[c, d] \subset[a, b], f$ is $H S$-integrable with respect to $g$ on $[c, d]$.

Proof. Let $\epsilon>0$ and $p \in \mathcal{P}(X)$. Then using Theorem 2.6, there exists a gauge $\delta_{p}$ on $[a, b]$ such that for all $\delta_{p}$-fine divisions $D_{1}$ and $D_{2}$ of $[a, b]$, $p\left(S\left[f, g, D_{1}\right]-S\left[f, g, D_{2}\right]\right)<\epsilon$. Let $Q_{1}$ and $Q_{2}$ be $\delta_{p}$-fine divisions of $[c, d]$. We consider the following cases:

Case 1. $a=c$ and $b=d$. This is obvious.

Case 2. $a=c$ and $d<b$. Let $Q$ be a $\delta_{p}$-fine division of $[d, b]$. Clearly, $D_{1}=Q_{1} \cup Q$ and $D_{2}=Q_{2} \cup Q$ are $\delta_{p}$-fine divisions of $[a, b]$. Now observe that

$$
\begin{aligned}
S\left[f, g, D_{1}\right]-S\left[f, g, D_{2}\right]= & S\left[f, g, Q_{1}\right]+S[f, g, Q] \\
& -\left(S\left[f, g, Q_{2}\right]+S[f, g, Q]\right) \\
= & S\left[f, g, Q_{1}\right]-S\left[f, g, Q_{1}\right] .
\end{aligned}
$$

Hence, $p\left(S\left[f, g, Q_{1}\right]-S\left[f, g, Q_{2}\right]\right)<\epsilon$.

Case 3. $a<c$ and $d=b$. This is similar to case 2 .

Case 4. $a<c$ and $d<b$. Let $R_{1}$ and $R_{2}$ be $\delta_{p}$-fine divisions of $[a, c]$ and [d,b], respectively. Clearly, $D_{1}=R_{1} \cup Q_{1} \cup R_{2}$ and $D_{2}=R_{1} \cup Q_{2} \cup R_{2}$ are $\delta_{p}$-fine divisions of $[a, b]$. Again observe that

$$
\begin{aligned}
S\left[f, g, D_{1}\right]-S\left[f, g, D_{2}\right]= & S\left[f, g, R_{1}\right]+S\left[f, g, Q_{1}\right]+S\left[f, g, R_{2}\right] \\
& -\left(S\left[f, g, R_{1}\right]+S\left[f, g, Q_{2}\right]+S\left[f, g, R_{2}\right]\right) \\
= & S\left[f, g, Q_{1}\right]-S\left[f, g, Q_{1}\right] .
\end{aligned}
$$

Hence, in any case $p\left(S\left[f, g, Q_{1}\right]-S\left[f, g, Q_{2}\right]\right)<\epsilon$. Thus, by Theorem 2.6, $f$ is HS-integrable with respect to $g$ on $[c, d]$.

\section{Some Existence Theorems}

This section presents some conditions for both the integrand and the integrator in order for the HS-integrals to exist. In what follows, $\chi_{A}$ denotes the characteristic function of $A \subset[a, b]$. Our first existence theorem depends on the following lemmas.

Lemma 3.1. Assume that $g \in G([a, b], X)$. Then $\chi_{\{a\}}$ is HS-integrable wrt $g$ on $[a, b]$. Moreover,

$$
(H S) \int_{a}^{b} \chi_{\{a\}} d g=g(a+)-g(a)
$$


Proof. Let $\epsilon>0$ and $p \in \mathcal{P}(X)$. Since $g(a+)$ exists, there exists $\delta^{\prime}>0$ such that $t \in\left(a, a+\delta^{\prime}\right) \Rightarrow p(g(t)-g(a+))<\epsilon$. Define a gauge $\delta$ on $[a, b]$ as follows. For each $t \in[a, b]$, let

$$
\delta(t)= \begin{cases}\frac{t-a}{2}, & \text { if } t \in(a, b] \\ \frac{\delta^{\prime}}{2}, & \text { if } t=a .\end{cases}
$$

Let $D=\left\{\left(\left[x_{i-1}, x_{i}\right], t_{i}\right)\right\}_{i=1}^{n}$ be a $\delta$-fine division of $[a, b]$. We know that

$$
t_{1} \in\left[a, x_{1}\right] \subset\left(t_{1}-\delta\left(t_{1}\right), t_{1}+\delta\left(t_{1}\right)\right) .
$$

If $t_{1} \neq a$, then $0<x_{1}-a<2 \delta\left(t_{1}\right)=t_{1}-a \leq x_{1}-a$ and we get a contradiction. Hence, $t_{1}=a$. Thus, $\chi_{\{a\}}\left(t_{1}\right)=1$ and $\chi_{\{a\}}\left(t_{2}\right)=\cdots=\chi_{\{a\}}\left(t_{n}\right)=0$. Observe that $a<x_{1}<t_{1}+\delta\left(t_{1}\right)=a+\delta(a)=a+\frac{\delta^{\prime}}{2}<a+\delta^{\prime}$. This implies that $x_{1} \in\left(a, a+\delta^{\prime}\right)$. Thus,

$$
\begin{aligned}
p\left(S\left[\chi_{\{a\}}, g, D\right]-[g(a+)-g(a)]\right) & =p\left(\chi_{\{a\}}\left(t_{1}\right)\left[g\left(x_{1}\right)-g(a)\right]-[g(a+)-g(a)]\right) \\
& =p\left(\left[g\left(x_{1}\right)-g(a)\right]-[g(a+)-g(a)]\right) \\
& =p\left(g\left(x_{1}\right)-g(a+)\right)<\epsilon .
\end{aligned}
$$

A similar result leads to the following lemma.

Lemma 3.2. Assume that $g \in G([a, b], X)$. Then $\chi_{\{b\}}$ is HS-integrable wrt $g$ on $[a, b]$. Moreover,

$$
(H S) \int_{a}^{b} \chi_{\{b\}} d g=g(b)-g(b-) .
$$

Lemma 3.3. Assume that $g \in G([a, b], X)$ and let $c \in(a, b)$. Then $\chi_{\{c\}}$ is $H S$-integrable wrt $g$ on $[a, b]$. Moreover,

$$
(H S) \int_{a}^{b} \chi_{\{c\}} d g=g(c+)-g(c-) .
$$

Proof. Clearly, $g$ is both regulated on $[a, c]$ and $[c, b]$. By Lemma 3.2,

$$
(H S) \int_{a}^{c} \chi_{\{c\}} d g=g(c)-g(c-) .
$$

By Lemma 3.1,

$$
(H S) \int_{c}^{b} \chi_{\{c\}} d g=g(c+)-g(c) .
$$

Hence,

$$
\begin{aligned}
(H S) \int_{a}^{b} \chi_{\{c\}} d g & =(H S) \int_{a}^{c} \chi_{\{c\}} d g+(H S) \int_{c}^{b} \chi_{\{c\}} d g \\
& =[g(c)-g(c-)]+[g(c+)-g(c)] \\
& =g(c+)-g(c-) .
\end{aligned}
$$


If we agree to set $g(a-)=g(a)$ and $g(b+)=g(b)$, then Lemmas 3.1, 3.2, and 3.3 yield

Lemma 3.4. Assume that $g \in G([a, b], X)$ and let $t \in[a, b]$. Then $\chi_{\{t\}}$ is $H S$-integrable wrt $g$ on $[a, b]$. Moreover,

$$
(H S) \int_{a}^{b} \chi_{\{t\}} d g=g(t+)-g(t-)
$$

Lemma 3.5. Assume that $g \in G([a, b], X)$. Then $\chi_{(a, b)}$ is HS-integrable wrt $g$ on $[a, b]$. Moreover,

$$
(H S) \int_{a}^{b} \chi_{(a, b)} d g=g(b-)-g(a+) .
$$

Proof. We have $\chi_{(a, b)}=1-\chi_{\{a\}}-\chi_{\{b\}}$. Thus,

$$
\begin{aligned}
(H S) \int_{a}^{b} \chi_{(a, b)} d g & =(H S) \int_{a}^{b} 1 \cdot d g-(H S) \int_{a}^{b} \chi_{\{a\}} d g-(H S) \int_{a}^{b} \chi_{\{b\}} d g \\
& =[g(b)-g(a)]-[g(a+)-g(a)]-[g(b)-g(b-)] \\
& =g(b-)-g(a+) .
\end{aligned}
$$

Lemma 3.6. Assume that $g \in G([a, b], X)$. Then for every $[c, d] \subset[a, b]$, $\chi_{(c, d)}$ is $H S$-integrable wrt $g$ on $[a, b]$. Moreover,

$$
(H S) \int_{a}^{b} \chi_{(c, d)} d g=g(d-)-g(c+) .
$$

Proof. We consider the following cases:

Case 1. $c=a$ and $d=b$. Using Lemma 3.5, we get

$$
(H S) \int_{a}^{b} \chi_{(c, d)} d g=(H S) \int_{c}^{d} \chi_{(c, d)} d g=g(d-)-g(c+) .
$$

Case 2. $c=a$ and $d<b$. Then

$$
\begin{aligned}
(H S) \int_{a}^{b} \chi_{(c, d)} d g & =(H S) \int_{a}^{d} \chi_{(c, d)} d g+(H S) \int_{d}^{b} \chi_{(c, d)} d g \\
& =(H S) \int_{c}^{d} \chi_{(c, d)} d g+(H S) \int_{d}^{b} 0 d g \\
& =g(d-)-g(c+) .
\end{aligned}
$$

Case 3. $a<c$ and $d=b$. Then

$$
\begin{aligned}
(H S) \int_{a}^{b} \chi_{(c, d)} d g & =(H S) \int_{a}^{c} \chi_{(c, d)} d g+(H S) \int_{c}^{b} \chi_{(c, d)} d g \\
& =(H S) \int_{c}^{d} 0 d g+(H S) \int_{c}^{d} \chi_{(c, d)} d g \\
& =g(d-)-g(c+) .
\end{aligned}
$$


Case 4. $a<c$ and $d<b$. Then

$$
\begin{aligned}
(H S) \int_{a}^{b} \chi_{(c, d)} d g & =(H S) \int_{a}^{c} \chi_{(c, d)} d g+(H S) \int_{c}^{d} \chi_{(c, d)} d g+(H S) \int_{d}^{b} \chi_{(c, d)} d g \\
& =(H S) \int_{a}^{c} 0 d g+(H S) \int_{c}^{d} \chi_{(c, d)} d g+(H S) \int_{d}^{b} 0 d g \\
& =g(d-)-g(c+) .
\end{aligned}
$$

We now state and prove the first existence theorem of this section.

Theorem 3.7. Assume that $g \in G([a, b], X)$. Then for every step function $\psi:[a, b] \rightarrow \mathbb{R}, \psi$ is HS-integrable wrt $g$ on $[a, b]$. Moreover, the value of $(H S) \int_{a}^{b} \psi d g$ can be calculated.

Proof. Write $\psi=\sum_{i=0}^{n} \hat{c}_{i} \chi_{\left\{x_{i}\right\}}+\sum_{i=1}^{n} c_{i} \chi_{\left(x_{i-1}, x_{i}\right)}$ where $\left\{\left[x_{i-1}, x_{i}\right]\right\}_{i=1}^{n}$ is some division of $[a, b]$ and $\hat{c}_{i}, c_{i} \in \mathbb{R}$ for $i=1, \ldots, n$. Applying Lemma 3.4, Lemma 3.6, and Theorem 2.4, we conclude that $\psi$ is HS-integrable wrt $g$ on $[a, b]$. Moreover,

$$
(H S) \int_{a}^{b} \psi d g=\sum_{i=0}^{n} \hat{c}_{i}\left[g\left(x_{i}+\right)-g\left(x_{i}-\right)\right]+\sum_{i=1}^{n} c_{i}\left[g\left(x_{i}+\right)-g\left(x_{i-1}-\right)\right]
$$

where we agreed that $g(a-)=g(a)$ and $g(b+)=g(b)$.

Theorem 3.8. Assume that $X$ is a complete LCS. If $f:[a, b] \rightarrow X$ is continuous on $[a, b]$ and $g:[a, b] \rightarrow \mathbb{R}$ is of bounded variation on $[a, b]$, then $f$ is $H S$-integrable with respect to $g$ on $[a, b]$. Moreover, for each $p \in \mathcal{P}(X)$,

$$
p\left((H S) \int_{a}^{b} f d g\right) \leq \sup _{t \in[a, b]} p(f(t)) \cdot T V_{a}^{b}(g)
$$

Proof. Let $q \in \mathcal{P}(X)$ and let $\epsilon>0$. Let $M=T V_{a}^{b}(g)$. Since $g$ is of bounded variation, $M$ is a nonnegative real number. Since $f$ is continuous, it follows that $f$ is uniformly continuous on $[a, b]$. Thus, there exists $\delta^{\prime}>0$ such that for all $x, y \in[a, b]$ with $|x-y|<\delta^{\prime}$, we have $q(f(x)-f(y))<\frac{\epsilon}{2(M+1)}$. Define a gauge $\delta$ on $[a, b]$ by $\delta(t)=\frac{\delta^{\prime}}{2}$ for all $t \in[a, b]$. Let $D_{1}$ and $D_{2}$ be any two $\delta$-fine divisions of $[a, b]$. Then we can choose a third $\delta$-fine division $D_{3}$ such that it is both finer than $D_{1}$ and $D_{2}$. Let $([u, v], t) \in D_{1}$ and suppose 
that $D_{u}^{v}=\left\{\left(\left[z_{i-1}, z_{i}\right], t_{i}\right)\right\}_{i=1}^{r}$ is the refinement of $([u, v], t)$ in $D_{3}$. Consider the difference $\Delta(u, v)=f(t)[g(v)-g(u)]-S\left[f, g, D_{u}^{v}\right]$. Now, we get

$$
\Delta(u, v)=\sum_{i=1}^{r}\left\{\left[f(t)-f\left(t_{i}\right)\right] \cdot\left[g\left(z_{i}\right)-g\left(z_{i-1}\right)\right]\right\} .
$$

Since $t \in[u, v]$ and $t_{i} \in\left[z_{i-1}, z_{i}\right] \subset[u, v] \subset(t-\delta(t), t+\delta(t))$ for all $i \in$ $\{1, \ldots, r\}$ we have $\left|t-t_{i}\right| \leq v-u<2 \delta(t)=\delta^{\prime}$. Thus for all $i \in\{1, \ldots, r\}$ we get $q\left(f(t)-f\left(t_{i}\right)\right)<\frac{\epsilon}{2(M+1)}$. Hence,

$$
\begin{aligned}
q(\Delta(u, v)) & \leq \sum_{i=1}^{r} q\left(\left[f(t)-f\left(t_{i}\right)\right] \cdot\left[g\left(z_{i}\right)-g\left(z_{i-1}\right)\right]\right) \\
& =\sum_{i=1}^{r}\left\{\left|g\left(z_{i}\right)-g\left(z_{i-1}\right)\right| \cdot q\left(\left[f(t)-f\left(t_{i}\right)\right]\right)\right\} \\
& <\sum_{i=1}^{r}\left\{\left|g\left(z_{i}\right)-g\left(z_{i-1}\right)\right| \cdot \frac{\epsilon}{2(M+1)}\right\} \\
& =\frac{\epsilon}{2(M+1)} \cdot \sum_{i=1}^{r}\left|g\left(z_{i}\right)-g\left(z_{i-1}\right)\right| \leq \frac{\epsilon T V_{u}^{v}(g)}{2(M+1)} .
\end{aligned}
$$

Note that since $D_{3}$ is finer than $D_{1}$, it follows that

$$
\sum_{([u, v], t) \in D_{1}} \Delta(u, v)=S\left[f, g, D_{1}\right]-S\left[f, g, D_{3}\right] .
$$

Thus,

$$
\begin{aligned}
q\left(S\left[f, g, D_{1}\right]-S\left[f, g, D_{3}\right]\right)=q\left(\sum_{([u, v], t) \in D_{1}} \Delta(u, v)\right) \\
\leq \sum_{([u, v], t) \in D_{1}} q(\Delta(u, v)) \\
\leq \frac{\epsilon}{2(M+1)} \cdot \sum_{([u, v], t) \in D_{1}} T V_{u}^{v}(g) \\
\quad=\frac{\epsilon}{2(M+1)} \cdot T V_{a}^{b}(g)=\frac{\epsilon M}{2(M+1)}<\frac{\epsilon}{2}
\end{aligned}
$$

By similar argument, $q\left(S\left[f, g, D_{3}\right]-S\left[f, g, D_{2}\right]\right)<\frac{\epsilon}{2}$. Hence,

$$
\begin{aligned}
q\left(S\left[f, g, D_{1}\right]-S\left[f, g, D_{2}\right]\right) \leq & q\left(S\left[f, g, D_{1}\right]-S\left[f, g, D_{3}\right]\right) \\
& +q\left(S\left[f, g, D_{3}\right]-S\left[f, g, D_{2}\right]\right) \\
< & \frac{\epsilon}{2}+\frac{\epsilon}{2}=\epsilon .
\end{aligned}
$$


Thus, by Theorem 2.6, the first part of the result follows.

For the last part, let $p \in \mathcal{P}(X)$ and $\eta>0$. Choose a gauge $\delta$ on $[a, b]$ such that $p\left(S[f, g, D]-(H S) \int_{a}^{b} f d g\right)<\eta$ whenever $D$ is a $\delta$-fine division of $[a, b]$. By hypothesis, $T V_{a}^{b}(g)<+\infty$. Fix a $\delta$-fine division $D=\left\{\left(\left[x_{i-1}, x_{i}\right], t_{i}\right)\right\}_{i=1}^{n}$ of $[a, b]$. Observe that

$$
\begin{aligned}
p(S[f, g, D]) & \leq \sum_{i=1}^{n} p\left(f\left(t_{i}\right)\left[g\left(x_{i}\right)-g\left(x_{i-1}\right)\right]\right) \\
& \leq \sum_{i=1}^{n} \sup _{t \in[a, b]} p(f(t)) \cdot\left|g\left(x_{i}\right)-g\left(x_{i-1}\right)\right| \\
& \leq \sup _{t \in[a, b]} p(f(t)) \cdot T V_{a}^{b}(g) .
\end{aligned}
$$

Hence,

$$
\begin{aligned}
p\left((H S) \int_{a}^{b} f d g\right) & \leq p\left((H S) \int_{a}^{b} f d g-S[f, g, D]\right)+p(S[f, g, D]) \\
& <\eta+\sup _{t \in[a, b]} p(f(t)) \cdot T V_{a}^{b}(g) .
\end{aligned}
$$

Because $\eta>0$ was arbitrary, the result follows.

\section{References}

[1] H. Jarchow, Locally Convex Spaces, B.G. Teubner, Stuttgart, 1981.

[2] Jong Sul Lim, Ju Han Yoon, and Gwang Sik Eun, On Henstock Stieltjes Integral, Kangweon-Kyungki Math. Journal 6(1)(1998), 87-96.

[3] V. Marraffa, Riemann type integral for functions taking values in a locally convex space, Czech. Math. J. 56(131)(2006), 475-489.

[4] W. Rudin, Functional Analysis, McGraw-Hill Inc, 1973.

[5] S. Schwabik, Abstract Perron-Stieltjes integral, Mathematica Bohemica, 121 (1996), No. 4, 425-447.

[6] T.J. Stieltjes, Recherches sur les fractions continues, Ann. Fac. Sci. Univ. Toulouse, 8 (1894), 1-122.

[7] R. Vyborny and Lee Peng Yee, The Integral: An Easy Approach after Kurzweil and Henstock, Australian Mathematical Society Lecture Series 14 Cambridge University Press, 2000.

Received: January 11, 2014 